\title{
Population spread in patchy landscapes under a strong Allee effect
}

\author{
Jeffrey Musgrave, Alexandre Girard \\ Department of Mathematics and Statistics \\ University of Ottawa \\ Ottawa ON, K16N5 \\ and \\ Frithjof Lutscher \\ Department of Mathematics and Statistics and Department of Biology \\ University of Ottawa \\ Ottawa ON, K16N5
}

January 2, 2015

\begin{abstract}
Many species of invasive insects establish and spread in regions around the world, causing enormous economical and environmental damage, in particular in forests. Some of these insects are subject to an Allee effect whereby the population must surpass a certain threshold in order to establish. Recent studies have examined the possibility of exploiting an Allee effect to improve existing control strategies. Forests and most other ecosystems show natural spatial variation, and human activities frequently increase the degree of spatial heterogeneity. It is therefore imperative to understand how the interplay between this spatial variation and individual movement behavior affects the overall speed of spread of an invasion. To this end, we study an integrodifference equation model in a patchy landscape and with Allee growth dynamics. Movement behavior of individuals varies according to landscape quality. Our study focuses on how the speed of the resulting traveling periodic wave depends on the interaction between landscape fragmentation, patch-dependent dispersal, and Allee population dynamics.
\end{abstract}




\section{Introduction}

The past decades have seen a significant increase of species invasions where introduced species have negative effects on local ecosystems, and a tendency to spread spatially. Mitigating or preventing these negative environmental and ecosystem effects can incur huge economic cost. A class of invasives that has attracted much attention recently are forest invasive insects that can devastate the natural environment, e.g. mountain pine beetle (Dendroctonus ponderosae), emerald ash borer (Agrilus planipennis), and the brown spruce longhorn beetle (Tetropium fuscum). Some of these species are subject to an Allee effect, whereby a newly introduced population must surpass a population threshold in order to establish (Courchamp et al., 2009). Recent studies have examined the possibility of exploiting such an Allee effect to improve control strategies for managing biological invasion, see Tobin et al. (2011) and references therein. As landscapes are heterogeneous, population growth rates and the strength of an Allee effect may vary spatially. Tobin et al. (2007) found such variation empirically for gypsy moth (Lymantria dispar) in North America. Spatial variation, combined with individual movement decisions in heterogeneous landscapes, may have a profound effect on the speed of spatial spread for an invasive organism. In this work, we study the interaction between individual dispersal behavior and Allee effect in patchy landscapes with the aim to aid future management strategies.

Frequently, the life cycle of forest insect populations consists of nonoverlapping, synchronous generations with well-defined growth and dispersal phases. Accordingly, we base our study on a mathematical multi-scale model, where we describe individual movement within the dispersal phase as a random walk in an infinite continuous patchy landscape and express the population dynamics during the growth phase via a discrete-time map. The resulting model is an integrodifference equation (IDE), consisting of a growth function and a dispersal kernel (Kot and Schaffer, 1986; Neubert et al., 1995).

The movement (sub-) model is formulated as a reaction-diffusion equation and includes individual behavior at habitat edges (Ovaskainen and Cornell, 2003; Maciel and Lutscher, 2013). In our previous work, we studied separately how dispersal kernels in patchy landscapes arise from such a model (Musgrave and Lutscher, 2013a), and how individual behavior affects persistence and spread in the absence of an Allee effect (Musgrave and Lutscher, 2013b). Here, we consider the situation with a strong Allee effect, i.e. the population declines at low density and experiences net growth only when its density is above the so-called Allee threshold (Courchamp et al., 2009). 
While IDEs are a highly popular modeling framework for spatial ecologists (Kot et al., 1996), their analysis in the presence of an Allee effect is still in its infancy (Kot et al., 1996; Veit and Lewis, 1996; Wang et al., 2002), and the combination between Allee effect and landscape heterogeneity is largely unexplored (but see Dewhirst and Lutscher (2009)). Veit and Lewis (1996) numerically analyzed an IDE with a strong Allee effect to model the population dynamics of the house finch in eastern North America. Kot et al. (1996) used a piecewise constant approximation to an Allee growth function and derived a formula for the speed of spread. Wang et al. (2002) generalized the work of Kot et al. (1996) by considering a general growth function satisfying the conditions for an Allee effect, and derived a formula for the sign of the speed.

In this manuscript, we analyze IDEs with a strong Allee effect in patchy landscapes. Our analysis focuses on the speed of a traveling (periodic) wave. Since the linear conjecture does not hold for a strong Allee effect, and since our dispersal kernels may not only depend on distance but explicitly on initial and final location, analytical results are out of reach at this point. Our approach is therefore based on carefully chosen numerical simulations. Our focus is on studying the interaction between landscape fragmentation, patch-dependent dispersal and Allee population dynamics.

In the next section, we introduce some theory of IDEs, including a more detailed analysis of a fairly general Allee growth function in Section 2.1 and the resulting invasion speeds for a simple dispersal kernel. In Section 3, we discuss how dispersal kernels appropriate for homogeneous and patchy landscapes may be derived from mechanistic movement models in the form of reaction-diffusion equations. In Section 4, we study an IDE with a strong Allee effect in patchy landscapes with homogeneous and patch-dependent dispersal behavior.

\section{Integrodifference equation model}

We consider a single population with discrete, non-overlapping generations with distinct growth and dispersal phases. Accordingly, population dynamics are modeled using a discrete-time, continuous-space model known as integrodifference equation (IDE). The most general form of an IDE is

$$
N_{t+1}(x)=\int_{\Omega} k(x, y) f\left(N_{t}(y), y\right) d y,
$$

where $N_{t}(x)$ denotes the population density at location $x \in \Omega$ in the $t^{\text {th }}$ generation, $\Omega$ is the region of biological interest, $f$ is a non-negative function 
that describes population growth during the sedentary phase, and $k$ is a dispersal kernel describing the outcome of movement during the dispersal phase. For fixed $y$, kernel $k(x, y)$ can be interpreted as the probability density of final location of an individual after dispersal, i.e. the product $k(x, y) \Delta x$ gives the probability that an individual who started its dispersal process at $y$ will settle in $[x, x+\Delta x$ ) (Neubert et al., 1995). We choose $\Omega=\mathbb{R}$ to represent a one-dimensional transect through the landscape.

There are several frequently-used dispersal kernels in homogeneous landscapes (Kot et al., 1996). For illustrations, we choose the Laplace kernel

$$
k(x, y)=\frac{a}{2} \exp (-a|x-y|)
$$

with mean dispersal distance $1 / a$ and variance $2 / a^{2}$. This kernel can be derived from a mechanistic movement model, and can therefore be generalized to patchy landscapes, as we will explain in Section 3.

\subsection{Population growth with Allee effect}

For the growth dynamics in a homogeneous landscape, we consider a generalization of the scaled Beverton-Holt function, namely

$$
f(N)=\frac{R N^{\gamma}}{1+(R-1) N^{\gamma}},
$$

with maximum per capita growth rate $R>1$ and shape parameter $\gamma>0$. For $\gamma=1$, we recover the Beverton-Holt function; for $\gamma=2$ and $R>2$, the function has a strong Allee effect (Wang et al., 2002). In general, the function has two fixed points $f(0)=0$ and $f(1)=1$. For a strong Allee effect in the general form, we require $f^{\prime}(0)<1$ and the existence of an intermediate fixed point $N_{a} \in(0,1)$.

The first condition requires $\gamma>1$, which actually implies $f^{\prime}(0)=0$. The intermediate fixed point, the Allee threshold $N_{a}^{*}$, satisfies

$$
(R-1) N^{\gamma}-R N^{\gamma-1}+1=0 .
$$

Point $N_{a}^{*}$ is in the required range only when $R$ is large enough. The critical case when $N=1$ is a double root occurs when $(\gamma-1) R-(R-1) \gamma=0$. This condition is satisfied when $R=\gamma$. Hence, we impose the conditions

$$
R>\gamma>1
$$

on the parameters for $f$ to have a strong Allee effect. As $\gamma$ increases, the Allee threshold $N_{a}^{*}$ increases, and when $\gamma=R$, the positive fixed points coincide. When $R<\gamma$, the fixed point $N_{a}^{*}>1$ is outside of the interval of interest. The population collapses for all initial conditions in $(0,1)$. 


\subsection{Spread in a homogeneous landscape}

The spatial dynamics of our model in a homogeneous landscape are well understood. If the dispersal kernel is symmetric and satisfies some exponential boundedness condition, then there exists a spreading speed, $c$, and a corresponding traveling wave solution (Weinberger, 1982). While there is no explicit formula for the spreading speed, its sign is given by the formula (Wang et al., 2002)

$$
\operatorname{sign}(c)=\operatorname{sign} \int_{0}^{1}[f(N)-N] d N .
$$

In particular, a population may advance $(c>0)$, retreat $(c<0)$ or localize in space $(c=0)$. These three scenarios are illustrated in Figure 1 (cf. Fig 2 in Wang et al. (2002)).

For our Allee function in (3), the wave speed increases with maximum growth rate $R$ and decreases as the Allee threshold increases, i.e. as $\gamma$ increases (left panels, Figure 2). We also illustrate the threshold conditions for which the population localizes, i.e. $c=0$ (right panel, Figure 2). The solid dot for $\gamma=2, R=3.2952$ corresponds to the situation considered by Wang et al. (2002).

\section{Movement Behavior and Dispersal Kernels in Patchy Landscapes}

To represent habitat heterogeneity, we consider a spatially periodically alternating landscape consisting of two types of patches, representing different habitat quality (Shigesada et al., 1986; Kawasaki and Shigesada, 2007). We denote by $l_{1}$ and $l_{2}$ the lengths of the different patch types, to which we refer as "good" and "bad", respectively. This terminology will correspond to the properties of the growth functions in those patches (Section 4). The spatial period is $l=l_{1}+l_{2}$; the fraction of $\operatorname{good}$ habitat is $p=\frac{l_{1}}{l}$. For a fixed value of $p$, large values of $l$ describe a landscape with few large good patches interspersed with large bad patches. The landscape is coarse grained, a typical dispersal event remains within a patch or crosses only one patch boundary, fragmentation is low (Dewhirst and Lutscher, 2009). Vice versa, when $l$ is small (for the same value of $p$ ) the landscape consists of many small good and bad patches, it is very finely grained. A typical dispersal event crosses many patches, and fragmentation is high.

In Musgrave and Lutscher (2013a), we proposed a generalization of the Laplace kernel in (2) to describe dispersal in such a strongly heterogeneous 

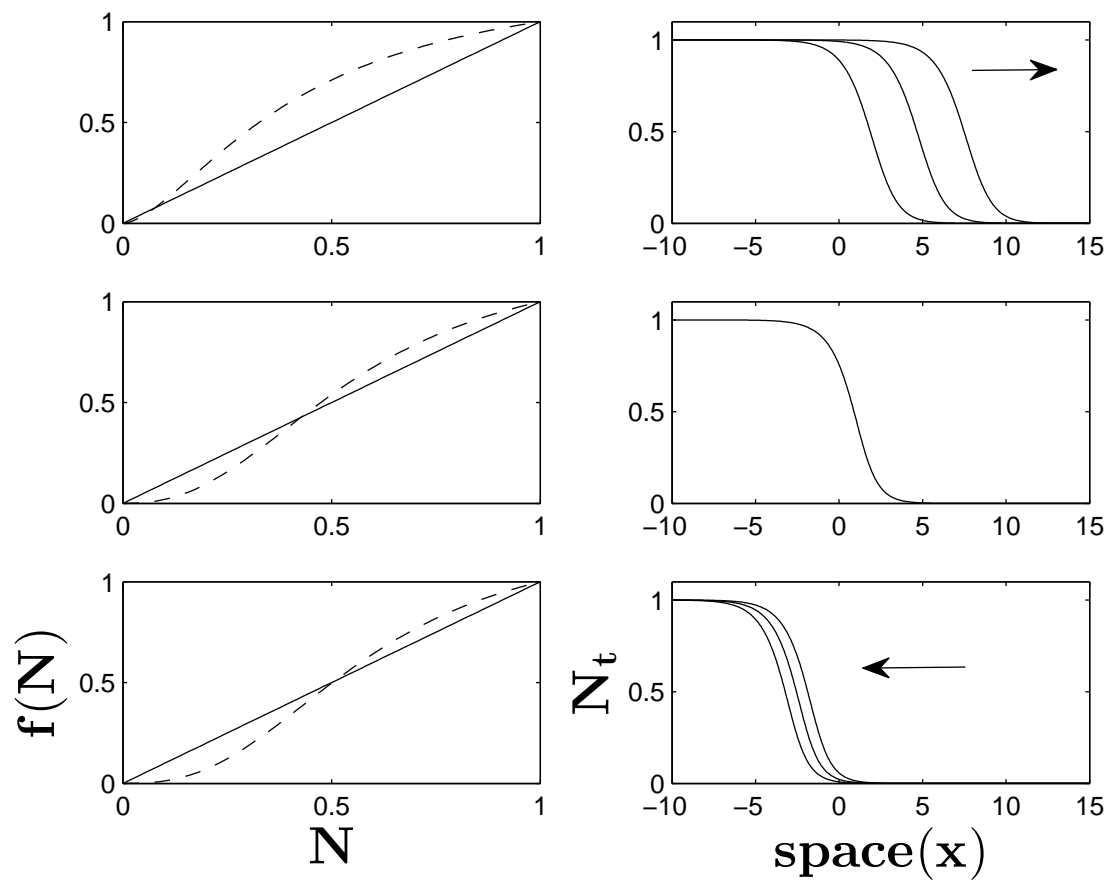

Figure 1: Plots of the reproduction function (dashed, left column) and corresponding spatial spread behavior (right column), illustrating the three possible outcomes of positive (top panel), zero (middle) or negative (bottom) wave speed according to formula (5). Parameters are $R=5$ and $\gamma=1.6$ (top panel), $\gamma=2.4$ (middle panel), and $\gamma=2.6$ (bottom panel). In all cases, the dispersal kernel is the Laplace kernel (2) with mean dispersal distance scaled to unity. 

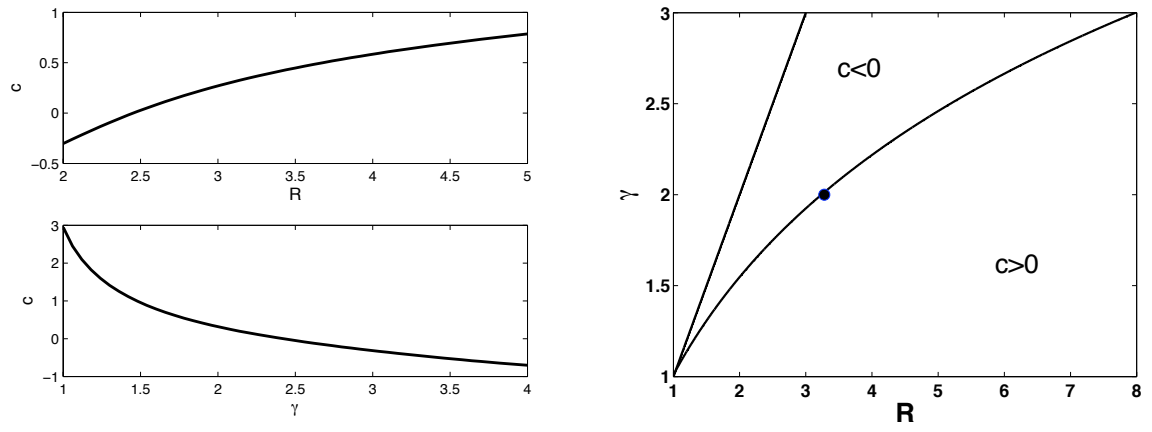

Figure 2: Left panel: Spreading speed in a homogeneous landscape as a function of the reproductive ratio $R$ (top graph) for fixed $\gamma=1.6$ and as a function of $\gamma$ (bottom graph) for fixed value $R=5$. Right panel: Level curve corresponding to $c=0$ in $R-\gamma$ parameter space. There are no traveling waves in the wedge $\gamma>R$. The solid dot on the curve corresponds to the point found by Wang et al. (2002) for $R \approx 3.29$ and $\gamma=2$.

landscape. We briefly summarize the approach here. We begin with the random walk model that leads to the Laplace kernel, according to Neubert et al. (1995).

Assume that an individual moves randomly on the real line for an exponentially distributed length of time. The density of this process satisfies the equation

$$
\frac{\partial u}{\partial T}=D \frac{\partial^{2} u}{\partial x^{2}}-\alpha u, \quad u(x, 0 ; y)=\delta(x-y),
$$

where $u(x, T ; y)$ is probability density of finding the individual at some location $x$ and time $T$, assuming that it started at $y$ at time zero. The parameters are the diffusion coefficient $D$ and the settling rate $\alpha$. The distribution of stopping locations for this process gives the resulting dispersal kernel. This distribution is obtained by integrating in time, for every fixed location $x$, the rate of an individual stopping. For the process described here, the formula

$$
k(x, y)=\int_{0}^{\infty} \alpha u(x, T ; y) d T
$$

results in the dispersal kernel (2) with parameter $a=\sqrt{\frac{\alpha}{D}}$ (Neubert et al., 1995). In the case of forest insects, we can also interpret $\alpha$ as the eggdeposition rate of a gravid female and $k$ as the distribution of eggs after dispersal of the gravid female. 
To generalize this approach to patchy landscapes, one assumes that an equation in the form of (6) holds on each patch, with parameters potentially varying between patches. Across patch boundaries, one chooses appropriate matching conditions (Ovaskainen and Cornell, 2003; Maciel and Lutscher, 2013). Specifically, the PDE system generalizing (6) is

$$
\frac{\partial u}{\partial T}(x, T ; y)=\left\{\begin{array}{l}
D_{1} \frac{\partial^{2} u(x, T ; y)}{\partial x^{2}}-\alpha_{1} u(x, T ; y), x \text { in good patches } \\
D_{2} \frac{\partial^{2} u(x, T ; y)}{\partial x^{2}}-\alpha_{2} u(x, T ; y), x \text { in bad patches. }
\end{array}\right.
$$

At the interface point, the flux of individuals must be continuous, but the density can be discontinuous if movement rates differ between patches and/or if individuals prefer one patch type over the other (Ovaskainen and Cornell, 2003; Maciel and Lutscher, 2013; Musgrave and Lutscher, 2013a). For example, if $x=0$ is an interface with a bad patch to the left and a good patch to the right, and if $(1+z) / 2$ and $(1-z) / 2$ are the probabilities that an individual at the interface moves to the right and left, respectively, then the interface conditions are

$$
\begin{aligned}
D_{2}(1+z) u\left(0^{-}, T ; y\right) & =D_{1}(1-z) u\left(0^{+}, T ; y\right) \\
D_{2} \frac{\partial u\left(0^{-}, T ; y\right)}{\partial x} & =D_{1} \frac{\partial u\left(0^{+}, T ; y\right)}{\partial x}
\end{aligned}
$$

where $z \in(-1,1)$ measures the bias towards the good $(z>0)$ or bad $(z<0)$ patch, and $0^{ \pm}$denotes the limit as $x$ approaches zero from the right and the left. Formula (7) can still be used to define the dispersal kernel resulting from (8) with (9) but is not accessible for explicit calculations. The dispersal kernel is, in general, not continuous with respect to $x$ and depends on $x, y$ explicitly rather than the distance $x-y$. In Figure 3 we illustrate the type of kernels that can result from (8) with (9), using five good patches. For a more detailed analysis of these kernels, see Musgrave and Lutscher (2013a).

\subsection{Numerical aspects}

The Laplace kernel and all kernels defined above for heterogeneous landscapes have unbounded support, yet computations can only be carried out on bounded domains, and kernels have to be truncated. In models without Allee effect, such truncations, in combination with stability issues, typically lead to numerical simulations predicting slower spread rates than could be expected from theoretical results. With a strong Allee effect, stability is typically not an issue, and truncation has only minor effects on numerical 


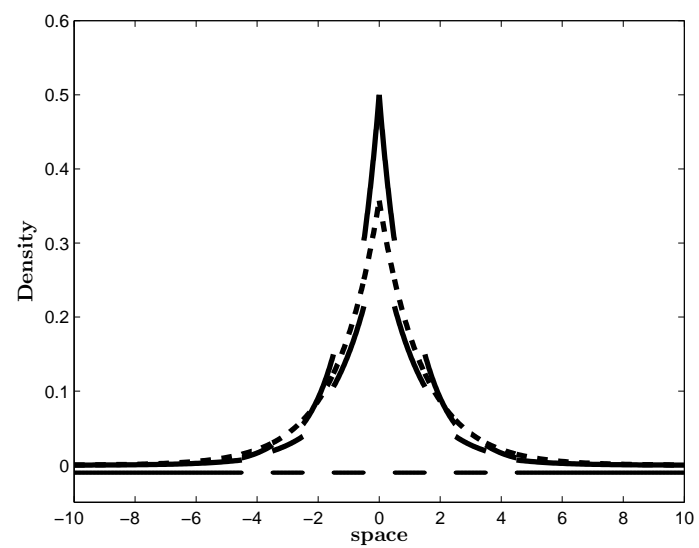

Figure 3: Dispersal kernels for a homogeneous (dashed) and heterogeneous (solid) dispersal process. The straight broken line at the bottom indicates habitat quality (solid black for bad patches, breaks for good patches). The dashed kernel is the Laplace kernel (2) that arises from $(8,9)$ for $D_{1}=D_{2}=$ $1, \alpha_{1}=\alpha_{2}=1$ and $z=0$. The solid curve has higher dispersal rates in bad patches $\left(D_{2}=2\right)$ and other parameters are identical.

spreading speeds since the strong Allee effect prohibits small population densities that arise in the (exponentially small) tails of the dispersal kernel from growing. We used the same numerical approach as in our previous work without Allee effect (Musgrave and Lutscher, 2013a). Specifically, we use kernels generated on five good patches, interspersed and surrounded by bad patches. We choose parameters such that less than $5 \%$ of individuals move beyond the range of these five good patches. We found that numerical results from this approach agreed very well with theoretical results in the absence of an Allee effect (Musgrave and Lutscher, 2013b). We therefore believe that the numerical results with strong Allee effect here are even closer to the true value (for which there is no theoretical expression as of yet). In addition, since the definition of $k$ in (7) is not computationally efficient, we rely on the equivalent Green's function approach that we developed earlier (Musgrave and Lutscher, 2013a).

Numerical integration of equation (1) requires that $\Omega$ is finite. We choose an interval so large compared to the dispersal scale that no boundary effects arise. We initialize the population as occupying the left half of the domain, and we simulate how the population spreads over many generations. Numerical methods for simulating equation (1) largely depend on the properties of 
the dispersal kernel. If dispersal is homogeneous, the kernel depends only on distance, e.g. the Laplace kernel (2), and the resulting convolution integral in (1) can be evaluated using MATLAB's fast Fourier transform ( $f f t$ ) routine. We used $2^{13}$ equidistant grid points on a domain of length $L=100$ and mean dispersal distance scaled to unity.

When dispersal is patch-dependent, we resort to standard techniques for numerical integration of equation (1), e.g. Simpson's Rule. We used again an equidistant grid with $2^{13}$ points, and, because of the discontinuity, we ensured that no patch interface falls on a grid point.

To determine the speed of population spread (or retreat) numerically, we follow Shigesada et al. (1986) and define the speed as the time averaged speed at which the population front is advancing or retreating. At the front of the wave, one defines a detection threshold, $\bar{N}$. We denote $\bar{x}(t)$ as the largest point at which the density satisfies $N_{t}(\bar{x}(t)) \geq \bar{N}$, so that $\bar{x}$ denotes the spatial location of the wave front. Assuming $\bar{x}(0)=0$, the average frontal speed is given by (Shigesada et al., 1986)

$$
\bar{c}=\lim _{t \rightarrow \infty} \frac{1}{t} \sum_{j=1}^{\infty}\left(\bar{x}\left(t_{j}\right)-\bar{x}\left(t_{j-1}\right)\right)=\lim _{t \rightarrow \infty} \frac{\bar{x}(t)}{t} .
$$

While the detection threshold $\bar{N}$ can be chosen fairly arbitrarily when there is no Allee effect, we found that with an Allee effect, the threshold needs to be chosen at least as large as the Allee threshold to obtain accurate results.

\section{Spread in a patchy landscape}

We model a strongly heterogeneous landscape by allowing the growth function $f(\cdot, y)$ to depend on patch type, as indicated in the second argument. We choose an extreme form, where, in a good patch, the growth function includes an Allee effect of the form in (3) with conditions (4) satisfied. A bad patch is completely hostile, so that we set $f=0$ there. We first consider the situation that dispersal is patch-independent, i.e. the dispersal kernel is the Laplace kernel. We distinguish the two cases of small versus large landscape period. In the former case, we use a homogenization argument, in the latter, we encounter a minimal domain-size problem and a gap-crossing problem. Later, we explore how patch-dependent movement alters the results obtained from homogeneous dispersal. 


\subsection{Homogeneous Dispersal, small period}

When dispersal is independent of landscape features, for example in winddispersed seeds, we continue to use the Laplace kernel with scaled average dispersal distance $a=1$. We are interested in how population spread depends on the percentage of good habitat $p=l_{1} / l$. When the period of the landscape is small, e.g. $l \ll 1$, previous work suggests that spatial averaging provides a good approximation of the spreading speed (Dewhirst and Lutscher, 2009).

The spatially averaged growth function is

$$
\bar{f}(N)=p \frac{R N^{\gamma}}{1+(R-1) N^{\gamma}}+(1-p) \times 0=p f(N) .
$$

The two positive fixed points corresponding to the carrying capacity $\left(N^{*}=\right.$ $\left.N^{*}(p)\right)$ and the Allee threshold $\left(N_{a}^{*}=N_{a}^{*}(p)\right)$ now depend on $p$. When $p$ is large enough, $N^{*}(p)$ is increasing in $p$ and $N_{a}^{*}(p)$ is decreasing in $p$. When $p$ is small, then there is no positive fixed point of $\bar{f}$, see Figure 4 . The critical point when $\bar{f}\left(N^{*}\right)=N^{*}$ and $\bar{f}^{\prime}\left(N^{*}\right)=1$ for $0<N^{*}<1$ occurs for

$$
p=p^{*}=\frac{\gamma}{R}\left(\frac{R-1}{\gamma-1}\right)^{\frac{\gamma-1}{\gamma}}
$$

Contour lines shown in Figure 5 illustrate the dependence of $p^{*}$ on $R$ and $\gamma$. Above each contour line, both $N_{a}^{*}$ and $N^{*}$ exist so that the population may persist if $N_{0}$ is large enough to overcome the Allee effect. However, below each line, the Allee effect is too strong and the population goes extinct as neither $N_{a}^{*}$ nor $N^{*}$ exist.

Accordingly, we expect that in the small-scale limit, a slight modification of formula (5) determines whether the population advances or retreats. Namely, provided that $N^{*}(p)>0$ we expect that the population advances if the integral

$$
\int_{0}^{N^{*}(p)}[\bar{f}(N)-N] d N
$$

is positive and retreats if this integral is negative. If $N^{*}(p)$ is not defined, we expect population collapse.

We illustrate the types of solutions that arise in a patchy landscape with an Allee effect depending on the fraction of good patches in Figure 6 . We choose parameters such that the population advances when $p=1$. As we decrease the percentage of good patches, the population slows down, localizes, then retreats, and eventually collapses. The latter behavior arises 


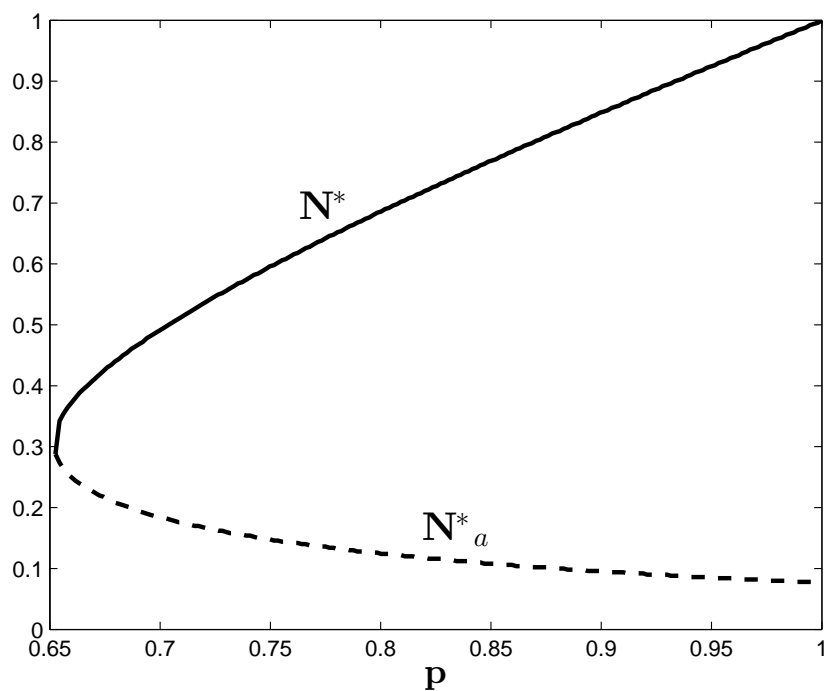

Figure 4: Carrying capacity and Allee threshold for the averaged growth function $\bar{f}$ in (11) as a function of the fraction of good patches $p$. Parameters are $R=5$ and $\gamma=1.6$.

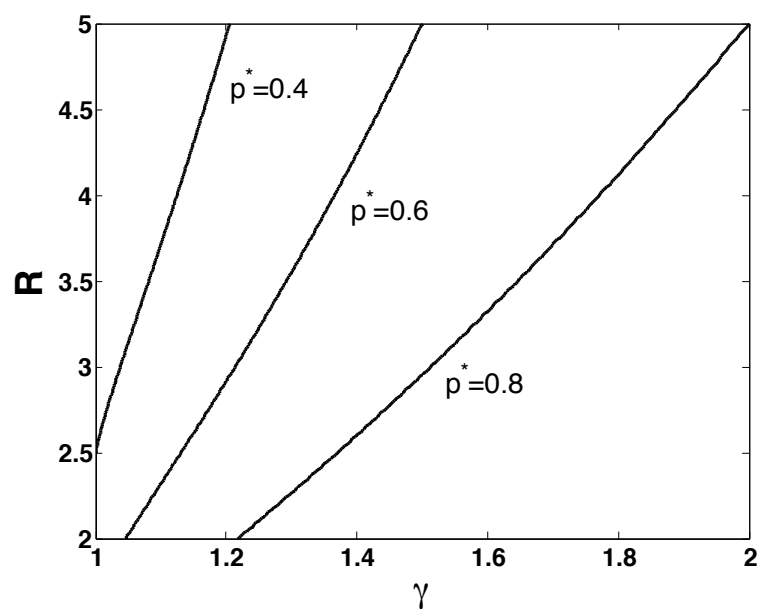

Figure 5: Dependence of the critical value $p^{*}$ for double roots on $R$ and $\gamma$. Above each contour line, both $N_{a}^{*}$ and $N^{*}$ exist so that persistence may occur whereas below each contour line the population goes extinct as the Allee effect is too strong. 
when the value of $p$ is so small that there are no positive fixed points of $\bar{f}$. The profile of the solution reflects the patchiness of the landscape: the population density is higher in good patches than in bad ones. Dispersal from good into bad patches prevents the population from going to zero there.

The rule of thumb in (13) turns out to be reasonably accurate even when $l$ is not very small. In Figure 7 we plot the numerically obtained speed of population advance and retreat as a function of $p$ for various values of $\gamma$. We find a good agreement (within 10\%) between the prediction of (13) and the numerical result. In general, the success of the invasion and the magnitude of the resulting speed of spread depends significantly on landscape fragmentation and the strength of the Allee effect. For example, in order for the population to localize or spread, good patches must account for at least $40 \%$ of the landscape when $\gamma=1.2$ whereas the percentage of good patches must increase to over $70 \%$ when $\gamma=1.6$. When the Allee effect is particularly strong (e.g. $\gamma=2.5$ ), the population retreats and/or dies out for all values of $p$ (not shown in plot).

\subsection{Homogeneous Dispersal, large period}

When the spatial period is large compared to the dispersal distance, a new phenomenon may arise. A bad patch may become so large that the population cannot cross it in sufficient numbers to reach the Allee threshold on the nearest good patch. Obviously, the population will not spread in that case. It may, however, localize if a good patch is large enough to support a population in isolation. Hence, we are interested in a minimum domain-size problem as well as a maximum gap-size problem. We study these two questions in more detail and use the results to understand the effects of spatial scales of habitat heterogeneity on invasion speeds.

We begin with the minimum domain-size question. On an isolated good patch of length $l_{1}$ a population can persist if there are enough individuals to begin with and if $l_{1}$ is large enough so that dispersal loss across the boundary is small. Because of the Allee effect, the conditions for existence of a positive stable equilibrium cannot be computed from the linearization at zero, and are, in general, not computable explicitly at all. Using the Laplace kernel, one can reduce the steady-state problem for the IDE to a secondorder ordinary differential equation (Kot and Schaffer, 1986). One can then use phase-plane and, depending on the growth function also energy methods, to obtain some explicit results. We continue our exploration numerically. We initialize the population at the non-spatial carrying capacity $N^{*}=1$ throughout the domain. We find numerically the value $l_{1}^{*}$ above which the 

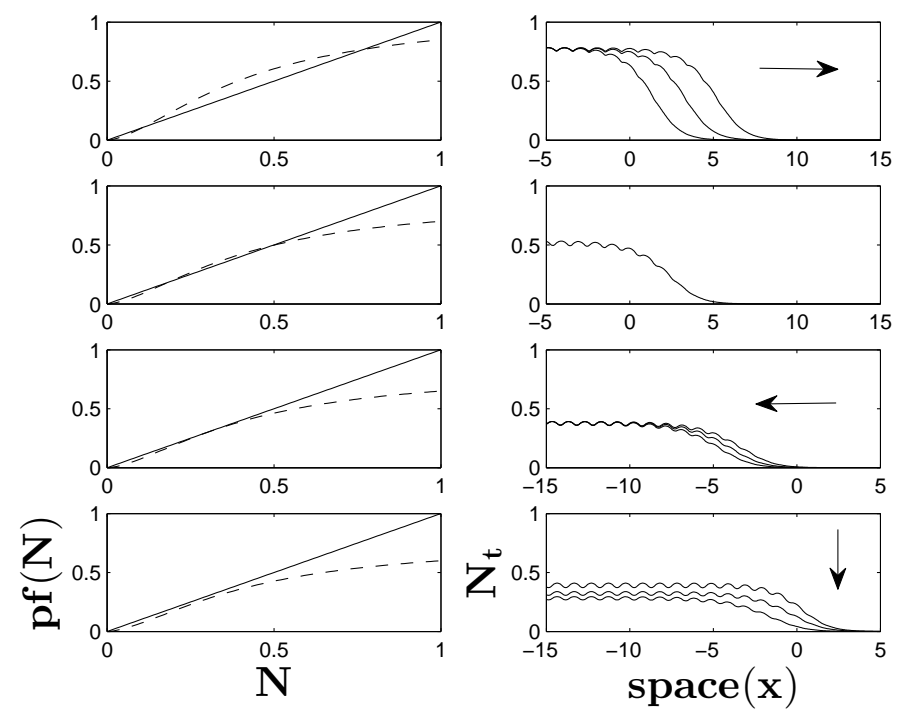

Figure 6: Numerical solution, for fifty generations, of equation (1) in a patchy landscape with $k$ given by a Laplace kernel with variance equal to unity. We chose $R=5$ and $\gamma=1.6$ which corresponds to the $c>0$ region in the right panel of Figure 2. First (top) panel: The area between the curve $p f(N)$ and the line $N$, up to the largest intersection $N^{*}(p)$, is positive which corresponds to the population advancing $(p=0.85)$. Second panel: The area between the curve $p f(N)$ and the line $N$ is zero which corresponds to localization $(p=0.7)$. Third panel: The area between the curve $p f(N)$ and the line $N$ is negative which corresponds to the population retreating $(p=0.65)$. Fourth panel: The curve $p f(N)$ lies completely below the line $N$ which corresponds to population extinction $(p=0.6)$. 


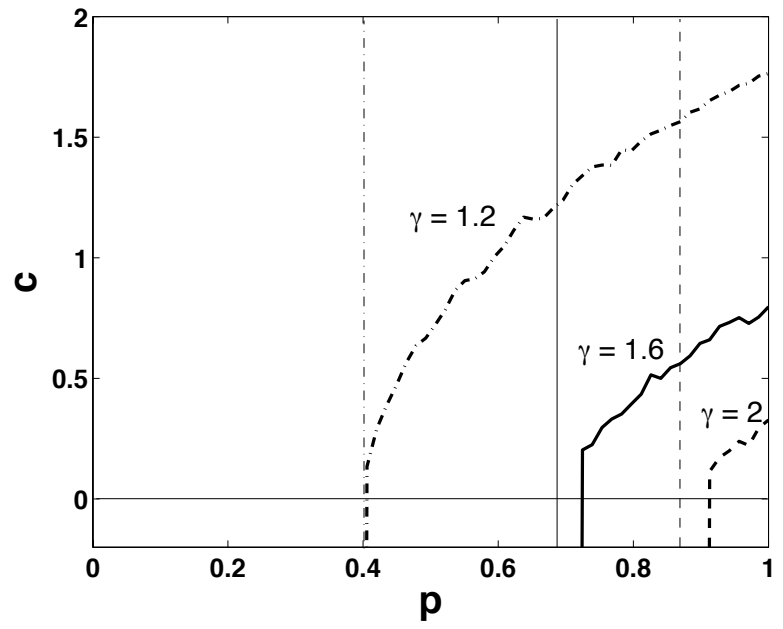

Figure 7: Spreading speed as a function of percentage of good habitat, $p$. The different lines correspond to different values of $\gamma=1.2$ (dash-dot), $\gamma=1.6$ (solid) and $\gamma=2$ (dashed). The vertical lines correspond to the values of $p$ for which the integral in (13) equals zero, which are $p^{*}=0.402, p^{*}=0.687$ and $p^{*}=0.87$, respectively. In all simulations, we chose the Laplace kernel with mean dispersal equal to unity, as well as $l=1$ and $R=5$. 

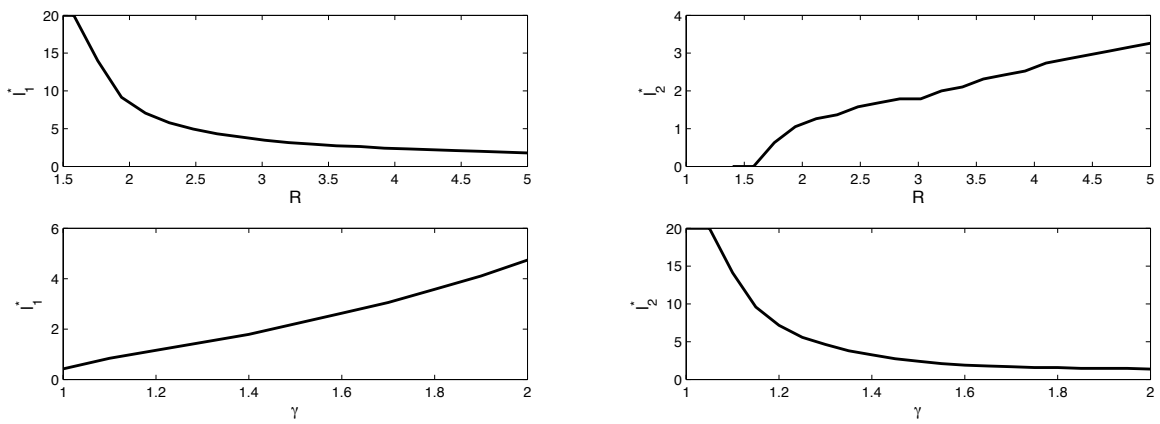

(a)

(b)

Figure 8: Left panel: Minimal good patch size $l_{1}^{*}$ decreases with growth rate $R$ and increases with shape parameter $\gamma$. Right panel: Maximal gap size $l_{2}^{*}$ increases with growth rate $R$ and decreases with shape parameter $\gamma$. Dispersal kernel is the Laplace kernel with mean dispersal distance scaled to unity. Fixed parameters are $R=5$ and $\gamma=1.4$.

population will reach a positive equilibrium and below which the population will go extinct. Figure 8(a) illustrates that $l_{1}^{*}$ decreases with growth rate $R$ and increases with shape parameter $\gamma$.

We now turn to the gap-crossing problem. We consider the extreme situation of two semi-infinite good patches separated by a hostile patch of length $l_{2}$. We initialize the population at $N_{0}=1$ on one side of the gap and $N_{0}=0$ on the other side, and we choose parameters of $f$ such that the population will spread in a homogeneously good habitat. When $l_{2}$ is small enough, sufficiently many individuals can cross the gap to start a population on the previously unoccupied patch; when $l_{2}$ is large, the density of dispersing individuals will not reach the Allee threshold and the population will not establish on the other side of the gap. We find numerically the maximum value $l_{2}^{*}$ that the population can cross. Dewhirst and Lutscher (2009) explored a similar idea for a simplified Allee function and three different dispersal kernels. Figure $8(\mathrm{~b})$ illustrates that $l_{2}^{*}$ increases with growth rate $R$ and decreases with shape parameter $\gamma$.

We now use these two threshold values for persistence and gap crossing to explain population spread and retreat or localization and collapse as the period of the landscape increases (i.e. when the homogenization approach from the previous section fails). Please note that the two thresholds $l_{1}^{*}, l_{2}^{*}$ depend only on the parameters $R, \gamma$ and $a$. For a given $p$, we then define the 
quantities

$$
l_{*}=\frac{l_{2}^{*}}{1-p}, \quad l_{* *}=\frac{l_{1}^{*}}{p} .
$$

If the landscape period, $l$, is larger than $l_{*}$ then the gaps are too large to cross. The population will not spread. If the landscape period is larger than $l_{* *}$ then each individual patch can support a population and hence there is no extinction or retreat. Altogether, we observe several cases. When $l>\max \left\{l_{*}, l_{* *}\right\}$ then the population will localize: it will neither spread nor go extinct from where it was introduced in sufficient numbers. When $l_{*}<l<l_{* *}$ the population may localize or go extinct. On the other hand, when $l_{* *}<l<l_{*}$ then the population may localize or spread. Finally, when $l<\min \left\{l_{*}, l_{* *}\right\}$, then all four possibilities may occur. Please note that $l_{*}$ is increasing in $p$ and $l_{* *}$ is decreasing.

We illustrate the behavior of the spreading speed as a function of landscape period in Figure 9. We choose growth parameters such that the population spreads in a homogeneous landscape $(R=5, \gamma=1.6)$ and choose $a=1$ in kernel $(2)$. We calculate $l_{1}^{*} \approx 2.65, l_{2}^{*} \approx 1.84$. Accordingly, for $p=0.5$, we obtain $l_{*} \approx 3.7<l_{* *} \approx 5.3$ (solid vertical lines in the figure). For this value of $p$, the population either localizes or collapses. For $p=0.8$, we find $l_{*} \approx 9.2>l_{* *} \approx 3.3$ (these thresholds are not plotted in the figure). Accordingly, the population spreads for a large range of $l$. For an intermediate value $p=0.7$, the thresholds are in the same order but closer together $\left(l_{*} \approx 6.1>l_{* *} \approx 3.8\right)$. The population spreads the fastest for intermediate values of $l$. For $p=0.6$ (with $l_{*} \approx 4.6>4.4 \approx l_{* *}$ ), we find that the population retreats when $l$ is too small.

In patchy landscapes, dispersal may hinder or help the invasion process depending on period of the landscape and the landscape fragmentation through parameter $p$. In the next section, we investigate how individual dispersal mechanisms in patchy landscapes influence the invasion process when an Allee effect is present.

\subsection{Patch-dependent dispersal behavior}

Most organisms have evolved behavioral responses to move through habitat of varying suitability. Foraging theory, for example, suggests that individuals should spend more time in high-quality patches than in low-quality patches, and they should move preferentially into high-quality patches. What effect, then, do such behavioral responses to patchiness have on the rate of spatial spread? 


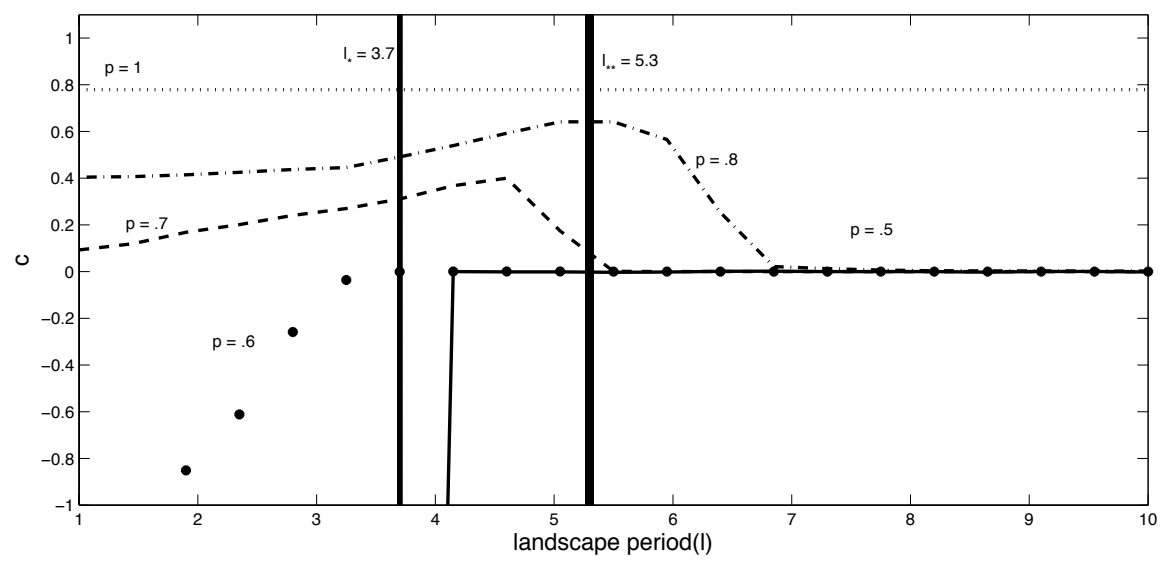

Figure 9: Spreading speed as a function of landscape period $l$ for various levels of fragmentation assuming $R=5$ and $\gamma=1.6$. The threshold values $l_{*}$ and $l_{* *}$ correspond to $p=0.5$; see main text.

The simulation result in Figure 10 demonstrates that adaptive movement behavior can make the difference between population collapse and spatial spread. For this figure, we chose parameters as in the bottom panel of Figure 6 so that the population collapses when dispersal is governed by the Laplace kernel (2) that arises from setting $D_{1}=D_{2}=1$ and $\alpha_{1}=\alpha_{2}=1$, so that the mean dispersal distance is equal to unity. Then we chose the dispersal rate in good patches to be lower than in bad patches (i.e. $D_{1}<D_{2}$ ) and generated a kernel from $(8,9)$. As a result, the population not only avoids collapse but even spreads spatially. With this motivation, we study the effect of the various movement parameters on the dynamics.

The consideration of patch-dependent dispersal behavior introduces five parameters instead of the mean dispersal distance for the Laplace kernel, namely $D_{1}, D_{2}, \alpha_{1}, \alpha_{2}$, and $z$ (see Section 3 ). We investigate the effect of individual parameters by perturbing from the homogeneous default setting $D_{1}=D_{2}=1, \alpha_{1}=\alpha_{2}=1$ and $z=0$, that results in the Laplace kernel (2) with mean dispersal distance equal to unity. We illustrate all our results by numerically evaluating the speed of population spread or retreat as a function of $p$, the fraction of good habitat. Unless explicitly otherwise noted in the figure caption, movement parameters are held at these default values.

We begin with the effect of patch preference, $z$, see Figure 11. When individuals choose to move into good habitat at a higher probability than 


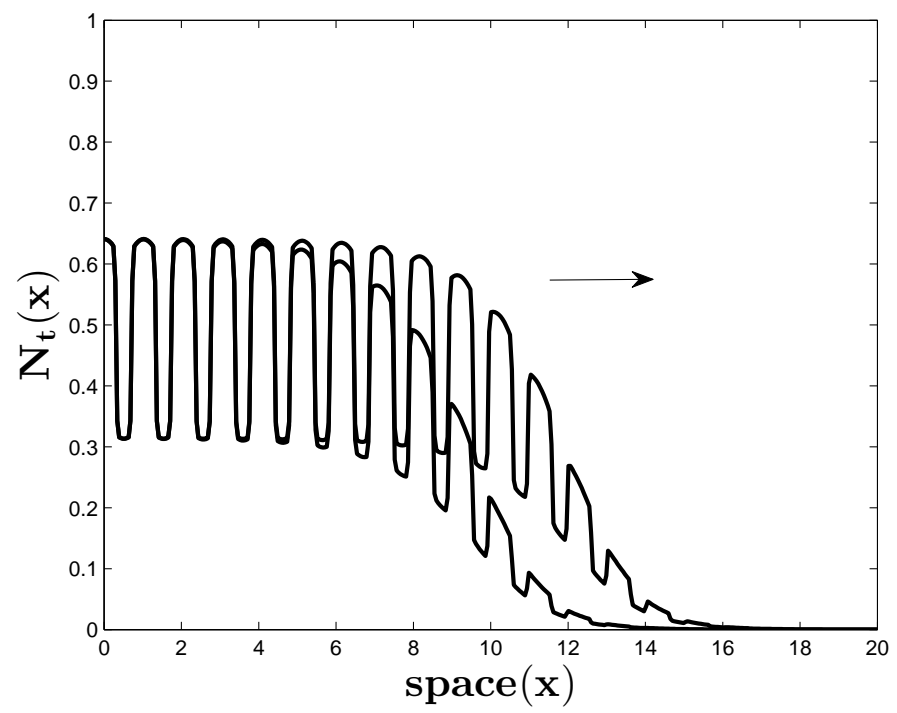

Figure 10: Numerical solution of equation (1) equipped with a dispersal kernel obtained from the individual dispersal model described in Section 3 with $D_{1}<D_{2}$. Growth parameters were chosen as in the bottom panel of Figure 6 corresponding to population collapse in a heterogeneous landscape with homogeneous dispersal. The spatial profile of the population now advances into a traveling periodic wave with positive speed. Parameter values are: $D_{1}=0.5, D_{2}=1, \alpha_{1}=\alpha_{2}=1, z=0, R=5, \gamma=1.6, p=0.6$, and $l=1$. 


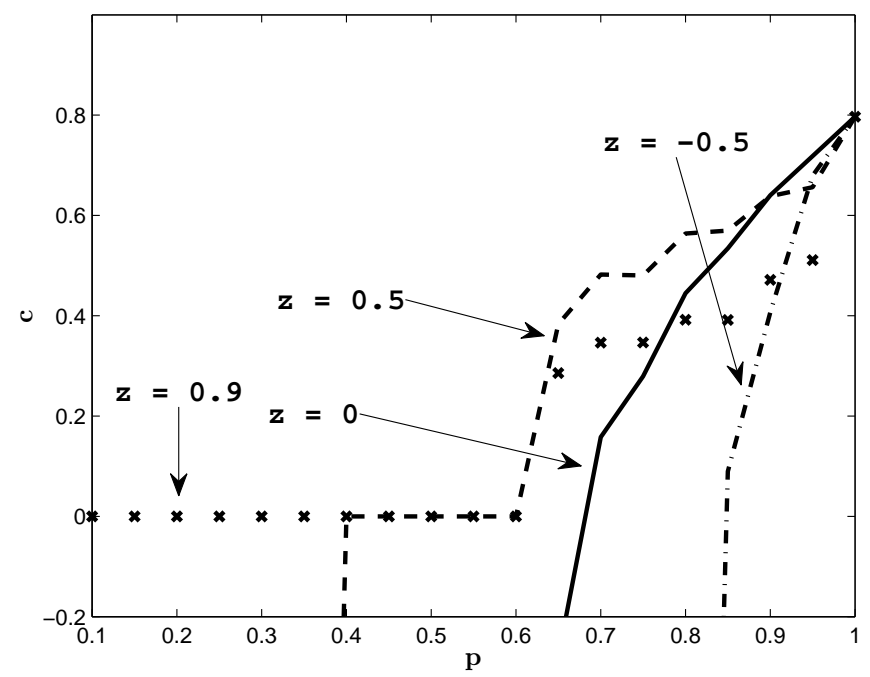

Figure 11: Spreading speed as a function of $p$ for different values of the effective movement bias. The solid line is the default of no movement bias $(z=0)$. When bad patches are preferred $(z<0$, dash-dot) the population slows or collapses. When good patches are preferred, the population spreads faster and for smaller percentage of good habitat $(z=0.5$, dashed). When preference for good patches is very strong $(z=0.9, \times \times)$, the population mostly localizes since only very few individuals leave a patch. Other parameters are $l=1, R=5$ and $\gamma=1.6$.

into bad habitat $(z>0)$, then the loss rate of individuals from good patches is reduced and the population can persist more easily. Accordingly, the spread rate can increase (e.g. $z=0.5$ ). However, increased preference for good patches also leads to decreased dispersal, so that for high $p$, the spread rate may decrease. For large values of $z$, this speed reduction is clearly visible (e.g. $z=0.9$ ) as is the extended region of localization for small values of $p$. In the extreme case of $z=1$ the population can persist on every good patch, independent of size but will not spread. When individuals preferentially move into bad patches $(z<0)$, overall mortality increases, since bad patches are hostile, and the invasion slows, halts or even collapses.

The diffusion rates in the two patch types have various effects on the spreading speed, see Figure 12. A higher diffusion rate in bad patches $\left(D_{2}=\right.$ 2 ) benefits the population in two ways. Individuals spend less time in bad 
patches and are therefore more likely to survive and reach the next good patch sooner. Hence, higher movement rates in bad patches lead to faster population spread whereas lower diffusion rates $\left(D_{2}=0.5\right)$ lead to lower spread rates as illustrated in panel (a). The dependence of the invasion speed on the movement rate in good patches is not monotone. When individuals move more slowly in good patches, they spend more time there and therefore have a higher reproductive output. On the other hand, the slower individual movement can slow overall population spread. When the percentage of good patches is high, a higher $D_{1}$ gives the higher spread rate, when the percentage of good patches is low, smaller values of $D_{1}$ are beneficial and lead to population persistence and spread, see panel (b).

Without any further illustration, we report that the spreading speed is a decreasing function of the settling rate in bad patches $\left(\alpha_{2}\right)$. Clearly, as more individuals settle in hostile patches, fewer are available to move the population forward. In fact, if $\alpha_{2}$ gets too large, the population will localize or even collapse. Similar to preference for good patches, the settling rate in good patches $\left(\alpha_{1}\right)$ has mixed effects on the invasion speed. If $\alpha_{1}$ is very small, the population will collapse, and an increase in $\alpha_{1}$ can prevent population extinction and allow spread. Very large values of $\alpha_{1}$, however, cause most individuals to stay within their native patch, so that population spread is impossible and the population localizes.

\section{Discussion}

Many invasive species are known worldwide, and new ones are discovered with increasing frequency. Since some of these species have enormous negative economic and ecological consequences, one would like to understand their dispersal mechanisms to devise appropriate containment strategies. Many invading species face an Allee effect due to factors such as mate finding (Taylor and Hastings, 2005), and these Allee effects can offer control options in addition to pesticide use (Blackwood et al., 2012). Spreading species also encounter habitats of different quality, from highly suitable to completely unsuitable. For example, many invasive forest insects are specialists that require a particular host species to lay eggs, for example mountain pine beetle, spruce budworm or pine sawyer. Natural variation in forest composition or human forest management can then generate situations where host trees are patchily distributed in a source-sink mosaic. In fact, silviculture practices, such as thinning of host trees, have been suggested as prevention measures against invasive forest insects. In Ontario, a $10 \mathrm{~km} \times 30 \mathrm{~km}$ large ash-free zone 


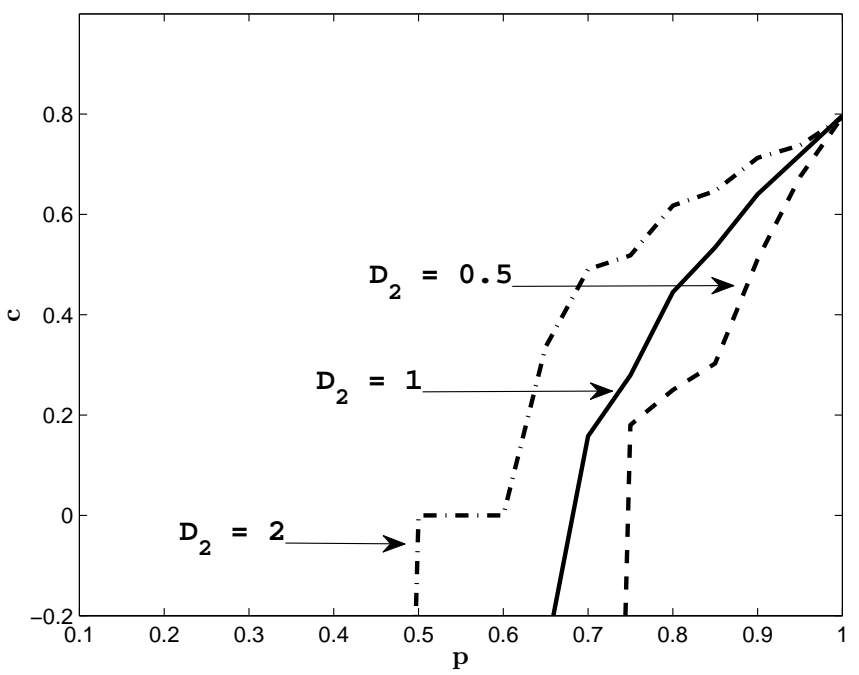

(a)

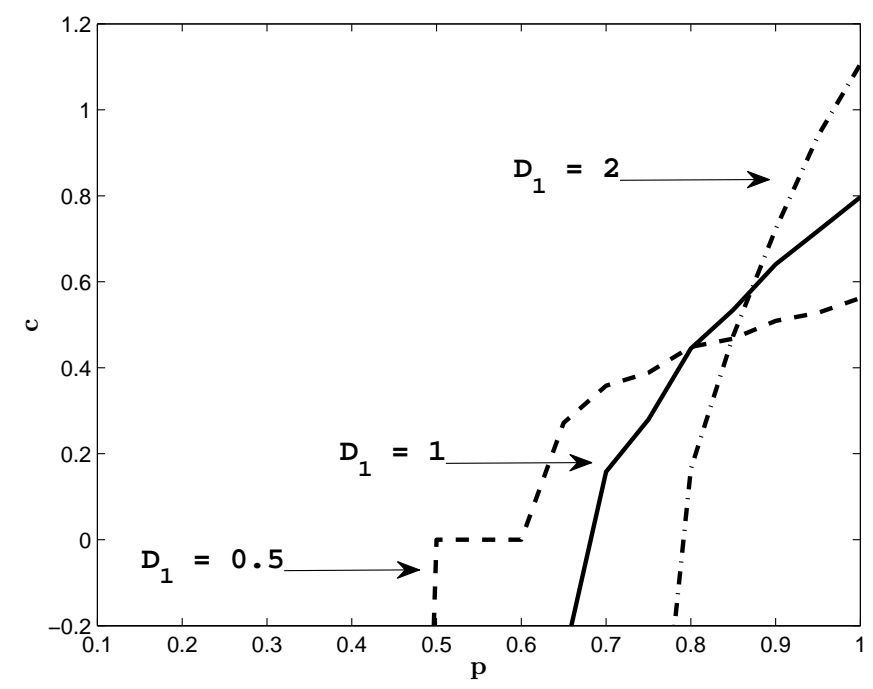

(b)

Figure 12: Panel (a): For all $p$, the spreading speed is a monotone increasing function of $D_{2}$ when $D_{1}=1$ is fixed. Panel (b): For large $p$ and fixed $D_{2}=1$, the spreading speed is higher when $D_{1}>1$ but for small $p$ the spreading speed is higher when $D_{1}<1$. The solid line corresponds to homogeneous dispersal as in the previous section. Fixed parameters are $l=1, R=5$ and $\gamma=1.6$. 
was established to halt the spread of emerald ash borer, but was unsuccessful. Clearly, before such large-scale measures are adopted, one would like to know how individuals move through heterogeneous landscapes so that one can determine the success or failure of a potential invasion (Kanarek et al., 2013).

In this work, we explored how individual-level movement behavior in a patchy landscape in combination with an Allee effect scales up to populationlevel spread rates. We considered the special case that bad patches are completely hostile, but our approach can be extended to situations where certain growth parameters vary in space. For example, there is empirical evidence that, the strength of the Allee effect in the gypsy moth varies regionally (Tobin et al., 2009), while there is a positive carrying capacity everywhere.

We used an integrodifference model for a population with discrete, nonoverlapping generations, and we generated appropriate dispersal kernels from an individual movement model in the form of a reaction-diffusion equation. In the absence of an Allee effect, fragmentation in integrodifference equations has been studied by a few authors (Weinberger, 2002; VanKirk and Lewis, 1997; Robbins, 2004; Kawasaki and Shigesada, 2007; Dewhirst and Lutscher, 2009). Our detailed description of movement behavior is based on recent advances in modeling random walks in such fragmented landscapes (Ovaskainen and Cornell, 2003; Musgrave and Lutscher, 2013a). Vice versa, spreading speeds for integrodifference equations with Allee effect in a homogeneous landscape are studied by Weinberger (1982); Kot et al. (1996); Veit and Lewis (1996); Wang et al. (2002). To our knowledge, this ours the first study of the combination of the two effects, but there is one example in Dewhirst and Lutscher (2009).

We discovered a few novel effects that arise from the interplay of fragmentation and Allee effect. In a homogeneous landscape, a population subject to an Allee effect will either spread or retreat. Localization occurs only for a highly specific combination of growth parameters, and is therefore empirically not relevant. In the patchy landscape, we find much larger regions in parameter space where localization occurs, and those regions depend on population growth and dispersal parameters, as well as landscape attributes. We also find that a population can collapse if the percentage of hostile patches is too large. From a management point of view, population collapse could be desired sometimes, but the percentage of hostile patches required for such a collapse may be too large to be feasible or even desirable. More importantly, the percentage of bad patches that will lead to population collapse depends on how individuals respond to fragmentation with their movement 
behavior. In fact, it is possible to increase the spread rate of a population by introducing hostile patches, provided that individuals move sufficiently fast through those hostile patches (Figure 12).

Our insights into conditions for localization of the population are closely related to a phenomenon known as propagation failure or range pinning (Lewis and Kareiva, 1993; Keitt et al., 2001). When space is modeled as discrete patches, a population may localize even if all patches are of equal good quality, provided that only relatively few individuals move from one patch to the next, so that the two stable steady states of the Allee effect can be maintained side-by-side. In those models, dispersal is modeled as a proportion of individuals moving from one patch to another. Our detailed study of several dispersal behaviors in a continuous landscape and their effect on range expansion versus range pinning gives a mechanistic explanation of these phenomena in terms of gap size and movement preferences.

There are a number of challenges that arise from our work, for example, one would like to have simple formulas, ideally modifications of equation (5) by Wang et al. (2002), to determine whether a population will expand, retreat, localize or collapse. In the case of small landscape period and homogeneous dispersal, the homogenization approach in (13) seemed successful. Clearly, the approach cannot work for large spatial periods, but recent results on reaction-diffusion equations for spread with Allee effect in patchy landscapes give hope that the homogenization technique can be successfully extended to heterogeneous dispersal (Maciel and Lutscher, submitted), and potentially also to larger spatial periods (Gilbert et al., 2014).

In patchy landscapes, individuals do adjust their movement behavior to local landscape attributes, and these behavioral adaptations can significantly affect the qualitative behavior of the dynamics of our model. For example, Figure 10 shows a traveling periodic wave with positive speed for the same parameter set that resulted in population extinction when dispersal behavior was not patch-dependent. Yet, these behavioral details are typically not included in theoretical models. Of course, gathering detailed information about such processes is very difficult, although some mark-recapture studies have focused on these issues (Safranyik et al., 1992; Schultz and Crone, 2001). The analysis of models that include these behavioral details is also still in its infancy. Some results are known for the case without Allee effect (Maciel and Lutscher, 2013; Musgrave and Lutscher, 2013b), but even then, some challenging analytical problems about the existence of a spreading speed and (discontinuous) traveling waves exist. In the presence of an Allee effect, where no explicit formulas for a wave speed are available even in a homogeneous landscape, we based our results completely on numerical 
solutions.

For more biological realism, the parameters in our model are almost certainly interdependent, whereas we varied each one individually. For example, the probability of staying inside a good patch could depend on the difference in habitat quality between a good and a bad patch, or on the distance to the next good patch. Also, not all individuals behave the same way so that a structured population model (e.g. by stage or age) would be more appropriate in many cases. A particularly interesting aspect could be a sex-structured model when dispersal behavior differs between male and female individuals. In such a system, movement strategies to overcome an Allee effect based on mate-limitation could be explored. Such work would greatly expand previous work on integrodifference equations and mate finding (Hurford et al., 2006) and sex-biased dispersal (Miller et al., 2011).

\section{Acknowledgments}

The authors wish to thank Gabriel Maciel for many insightful discussions. JM gratefully acknowledges graduate scholarship from the Natural Science and Engineering Research Council (NSERC) of Canada. AG was partially supported by the work-study program at the University of Ottawa. FL is supported by a Discovery grant from NSERC.

\section{References}

Blackwood, J., Berec, L., Yamanaka, T., Epanchin-Niell, R., Hastings, A., Liebhold, A. 2012. Bioeconomic synergy between tactics for insect eradication in the presence of allee effects. Proceedings of the Royal Society of London B 279, 2807-2815.

Courchamp, F., Berec, L., Gascoigne, J.. Allee Effects in Ecology and Conservation. OUP, Oxford, United Kingdom 2009.

Dewhirst, S., Lutscher, F. 2009. Dispersal in heterogeneous habitats: Thresholds, spatial scales, and approximate rates of spread. Ecology 90, $1338-1345$.

Gilbert, M., White, S., Bullock, J., Gaffney, E. 2014. Spreading speeds for stage structures plant populations in fragmented landscapes. Journal of Theoretical Biology 349, 135-149. 
Hurford, A., Hebblewhite, M., Lewis, M. 2006. A spatially explicit model for an allee effect: why wolves recolonize so slowly in greater yellowstone. Theoretical Population Biology 70, 244-254.

Kanarek, A., Webb, C., Barfield, M., Holt, R. 2013. Allee effects, aggregation, and invasion success. Theoretical Ecology 6, 153-164.

Kawasaki, K., Shigesada, N. 2007. An integrodifference model for biological invasions in a periodically fragmented environment. Japan Journal of Industrial and Applied Mathematics 24, 3-15.

Keitt, T., Lewis, M., Holt, R. 2001. Allee effects, invasion pinning, and species' borders. American Naturalist 157(2), 203-216.

Kot, M., Lewis, M., van den Driessche, P. 1996. Dispersal data and the spread of invading organisms. Ecology 77, 2027-2042.

Kot, M., Schaffer, W.M. 1986. Discrete-time growth models. Mathematical Biosciences 80, 109-136.

Lewis, M., Kareiva, P. 1993. Allee dynamics and the spread of invading organisms. Theoretical Population Biology 43, 219-233.

Maciel, G., Lutscher, F. submitted. Allee effects and population spread in patchy landscapes. Journal of Biological Dynamics

Maciel, G.A., Lutscher, F. 2013. How how individual movement response to habitat edges affects population persistence and spatial spread. The American Naturalist DOI 10.1086/670661

Miller, T., Shaw, A., Inouye, B., Neubert, M. 2011. Sex-biased dispersal and the speed of two-sex invasions. The American Naturalist 5, 549-561.

Musgrave, J., Lutscher, F. 2013a. Integrodifference equations in patchy landscapes I. Dispersal kernels. Journal of Mathematical Biology DOI $10.1007 / \mathrm{s} 00285-013-0714-2$

Musgrave, J., Lutscher, F. 2013b. Integrodifference equations in patchy landscapes II. Population level consequences. Journal of Mathematical Biology DOI 10.1007/s00285-013-0715-1

Neubert, M., Kot, M., Lewis, M. 1995. Dispersal and pattern formation in a discrete-time predatory-prey model. Theoretical Population Biology 48, $7-43$. 
Ovaskainen, O., Cornell, S.J. 2003. Biased movement at a boundary and conditional occupancy times for diffusion processes. Journal of Applied Probability 40, 557-580.

Robbins, T.. Seed dispersal and biological invasions: A mathematical analysis. Ph.D. thesis, University of Utah, Salt Lake City, Utah 2004.

Safranyik, L., Linton, D., Silversides, R., McMullen, L. 1992. Dispersal of released mountain pine beetles under the canopy of a mature lodgepole pine stand. Journal of Applied Entomology 113, 441-450.

Schultz, C., Crone, E. 2001. Edge-mediated dispersal behavior in a prairie butterfly. Ecology 82(7), 1879-1892.

Shigesada, N., Kawasaki, K., Teramoto, E. 1986. Travelling periodic waves in heterogeneous environments. Theoretical Population Biology 30, 143160 .

Taylor, C., Hastings, A. 2005. Allee effects in biological invasions. Ecology Letters 8, 895-908.

Tobin, P., Berec, L., Liebhold, A. 2011. Exploiting allee effects for managing biological invasions. Ecology Letters 14, 615-624.

Tobin, P., Robinet, C., Johnson, D., Whitmire, S., Bjørnstad, O., Liebhold, A. 2009. The role of allee effects in gypsy moth, Lymantria dispar (1.), invasions. Population Ecology 51, 374-383.

Tobin, P., Whitmire, S., Johnson, D., Bjørnstad, O., Liebhold, A. 2007. Invasion speed is affected by geographical variation in the strength of allee effects. Ecology Letters 10, 36-43.

VanKirk, R.W., Lewis, M.A. 1997. Integrodifference models for persistence in fragmentated habitats. Bulletin of Mathematical Biology 59, 107-137.

Veit, R., Lewis, M. 1996. Dispersal, population growth, and the allee effect, dynamics of the house finch invasion of eastern north america. American Naturalist 148, 83-97.

Wang, M.H., Kot, M., Neubert, M. 2002. Integrodifference equations, allee effects, and invasions. Journal of Mathematical Biology 44, 150-168.

Weinberger, H.F. 1982. Long-time behavior of a class of biological models. SIAM Journal of Mathematical Analysis 13, 353-396. 
Weinberger, H.F. 2002. On spreading speeds and traveling waves for growth and migration models in a periodic habitat. Journal of Mathematical Biology 45, 511-548. 